\title{
Vagus Nerve Paraganglioma
}

National Cancer Institute

\section{Source}

National Cancer Institute. Vagus Nerve Paraganglioma. NCI Thesaurus. Code C8427.

A benign or malignant extra-adrenal parasympathetic paragang lioma arising from parag ang lia in the vagus nerve. Patients may present with a slow growing, painless mass in the neck, hoarseness, vocal cord paralysis, and dysphagia. 